\title{
Qualitative Behavior of AIDS in a Homosexual Population
}

\author{
Pedro Pablo Cárdenas Alzate ${ }^{1}$, Diana Paola Vasquez $\mathrm{S}^{2} \&$ Luz Enith Velasquez $\mathrm{R}^{2}$ \\ ${ }^{1}$ Department of Mathematics and GEDNOL, Universidad Tecnológica de Pereira, Colombia \\ ${ }^{2}$ Fundación Universitaria del Área Andina Seccional - Pereira, Colombia \\ Correspondence: Pedro Pablo Cárdenas Alzate, Department of Mathematics, Universidad Tecnológica de Pereira, \\ Pereira R, Colombia.
}

Received: November 14, 2019

Accepted: December 4, 2019

Online Published: December 5, 2019

doi:10.5539/mas.v14n1p1

URL: https://doi.org/10.5539/mas.v14n1p1

\begin{abstract}
The main idea of this work is to build a mathematical model that provides a better understanding of the qualitative behavior of AIDS in a male homosexual population. This model initially assumes a homogeneous combination taking three possibilities of clinical cases: Seropositive, full AIDS and the associated complex relationship (ARC). The proposed mathematical model is described by a dynamic system of nine differential equations which involves nine unknowns.
\end{abstract}

Keywords: AIDS, ARC, propagation of AIDS, seropositive

\section{Introduction}

At the end of the 70s, a group of doctors in the city of New York registered an unusual number of cases of Kaposi's sarcoma. This is an unknown and cancerous disease was made known in a large male population of homosexuals. This was an essential element of what we now call the century's epidemic: Acquired immunodeficiency syndrome (AIDS).

The human immunodeficiency virus (HIV) is a lymphotropic retrovirus, which can in many cases cause a wide range of immunological disturbances and thus the victim becomes susceptible to a number of infections and opportunistic cancers, which take advantage of the opportunity of the Low resistance to infect and destroy.

Among the unknown epidemiological factors are the duration of the latent period of infection (the time from infection to the point when the sick person is infectious to other members of the population), the proportion of infected persons who will exhibit any of the clinical forms of infection the disease as will be seen later, the duration of viral persistence within the victim after infection and the infectious period.

A patient infected with HIV can follow one of three paths: Full AIDS, this is a well-defined nosological entity; the socalled complex relationship associated with AIDS (ARC), a syndrome characterized by a lindadenopathy and some signs of AIDS disease. Finally, a person infected with HIV can remain seropositive for life without presenting any of the disease.

To be diagnosed as ARC, it is clear that the patient must present a symptom, a sign and an immunological abnormality (laboratory) which is presented below. In addition to this, the patient should be free of tumor-type opportunistic infections.

\section{Symptom}

- Severe discomfort and inaction

- Weight loss (more than $10 \%$ of body weight)

- Unexplained diarrhea greater than one month

- Unexplained fever

- $\quad$ Night sweats

\section{Clinical signs}

- Oral leukoplakia

- Oral candidiasis (fungi) 
- Persistent generalized lymphadenopathy (PGL)

- Spleen enlargement

- Skin rashes, exzemas, seborrhea and folliculitis

\section{Abnormal laboratory results}

- $\quad$ Lymphocytopenia $\left(<1.5 \times 10^{9} / L\right)$

- Thrombocytopenia $\left(<150 \times 10^{9} / L\right)$

- Anemia

- Exhaustion of T cells (defense)

- Low response of T-lymphocytes to mitogenic stimulation

- Increase in serum immunoglobulin levels

\section{Method}

\subsection{Model Description}

The mathematical model presented below is based on a type of Bailey model (Bailey, 1975) for sexually transmitted infections and the model of Anderson and May. It is important to note that here difference is made with these models since we have incorporated the ARC clinical status proposed by Massad (Massad, 1989).

We initially assume a homosexual population of size $P(t)$ comprising 8 types of individuals:

- $\quad X(t)$ is the density of susceptible individuals that will be infected with a transmission efficiency $b$.

- $y_{1}(t), y_{2}(t)$ and $y_{3}(t)$ are three types of individuals infected with HIV. This depends on the clinical state that will develop after a certain incubation period $\frac{1}{r}$.

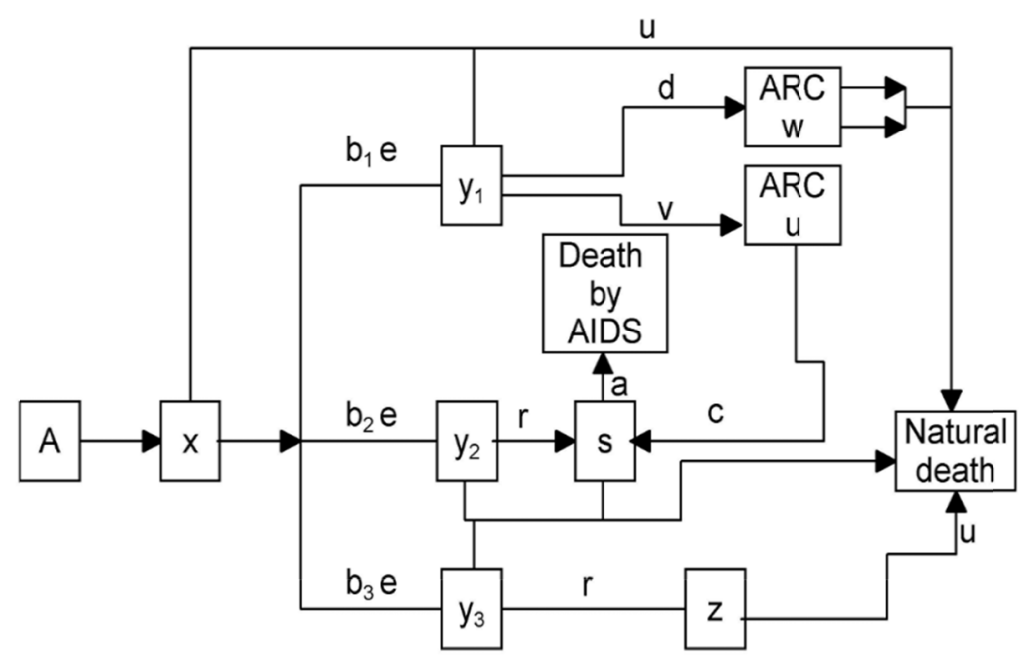

Figure 1. Flows between the compartments in the model

Now, three possible clinical forms:

- $\quad[W(t)+U(t)]$ ARC individuals

- $S(t)$ individuals with AIDS

- $\quad Z(t)$ seropositive individuals

In Figure 1, we can see the biological assumptions.

\subsection{Equations}

The model that describes the dynamic transmission of the HIV virus within the homosexual population, where $\frac{Y(t)}{P(t)}$ expresses the probability of any encounter with an infected individual and the net transmission rate is 
proportional to the product $X(t) \frac{Y(t)}{P(t)}$, which can be deduced from Figure 1 in the normal form of a system of differential equations:

$$
\begin{aligned}
& \frac{d P}{d t}=A-u P-a S \\
& \frac{d X}{d t}=A-\left(b_{1} e+b_{2} e+b_{3} e\right) X \frac{Y}{P}-u Y \\
& \frac{d y_{1}}{d t}=b_{1} e X \frac{Y}{P}-(u+d+v) y_{1} \\
& \frac{d y_{2}}{d t}=b_{2} e X \frac{Y}{P}-(u+r) y_{2} \\
& \frac{d y_{3}}{d t}=b_{3} e X \frac{Y}{P}-(u+r) y_{3} \\
& \frac{d W}{d t}=d y_{1}-u W \\
& \frac{d U}{d t}=v y_{1}-(u+c) U \\
& \frac{d S}{d t}=r y_{2}+c u-(u+a) S \\
& \frac{d Z}{d t}=r y_{3}-u Z
\end{aligned}
$$

This is a dynamic system of nine differential equations (autonomous) where:

- $\quad e$ represents the average number of acquisition of new sexual partners per unit of time.

- $b=1$ represents the probability that an infected homosexual transmits HIV to their partner during intercourse for each class of infected $y i$, donde $b=\sum_{i} b_{i}$.

- $A$ is the reason for immigration of susceptible persons into the homosexual community.

- $u$ is the reason for natural death (in the absence of the disease, the size of the population in steady state is $P^{*}=\frac{A}{u}$ of the community.

- $\quad a$ is the reason for death of AIDS patients.

- $\frac{1}{r}, \frac{1}{d}, \frac{1}{v}$ are incubation periods (the interval between the time of HIV infection and the time in which the signs of full AIDS are given), where $d+v=r ; r, d, v$, determine the reasons for the development of the infection.

Now, equilibrium populations are (Cardenas, 2017):

$$
\begin{gathered}
P^{*}=\frac{\operatorname{Abe}\left(b e(u+a)-[a /(u+a)]\left\{r b_{2} e-v[1+u /(u+c)]\right\} b_{1} e\right)}{t} \\
X^{*}=\frac{u+r}{b e} P^{*}
\end{gathered}
$$




$$
\begin{aligned}
Y^{*} & =\frac{A}{u+r}-\frac{u}{b e} P^{*} \\
W^{*} & =\frac{d b_{1} e}{u(u+r)}-\left(\frac{A b e-u(u+r)}{(b e)^{2}} P^{*}\right) \\
U^{*} & =\frac{v b_{1} e X^{*} Y^{*}}{(u+c)(u+r) P^{*}} \\
S^{*} & =A-\frac{u P^{*}}{a} \\
Z^{*} & =\frac{r b_{3} e}{u(u+r)}\left(\frac{A b e-u(u+r) P^{*}}{(b e)^{2}}\right)
\end{aligned}
$$

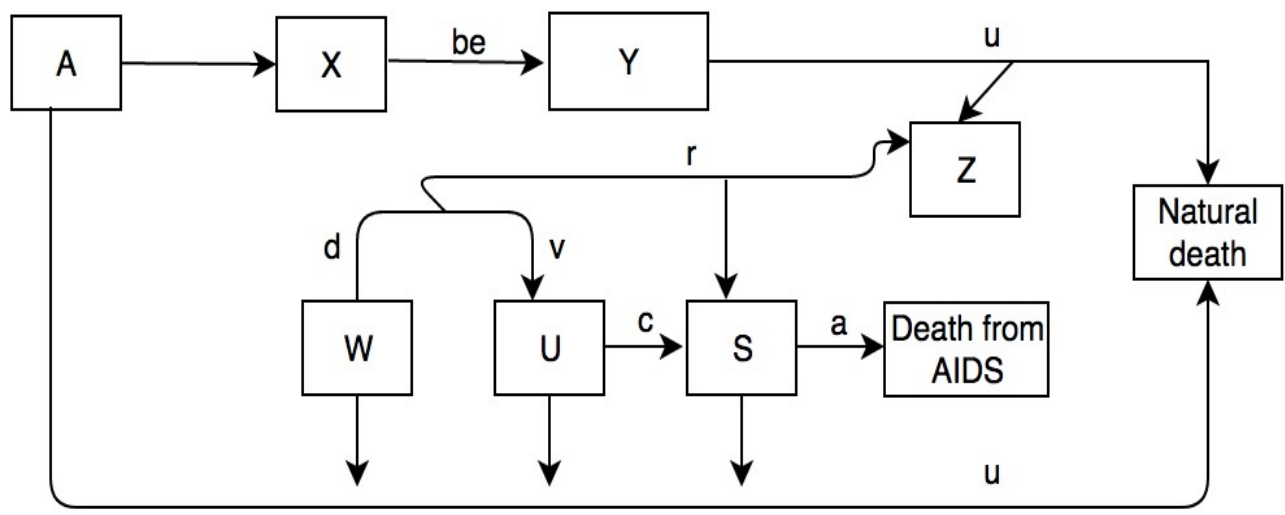

Figure 2. Simplification of the diagram in figure 1

Therefore, with the assumption that

$$
\begin{gathered}
Y(t)=y_{1}(t)+y_{2}(t)+y_{3}(t) \\
b=b_{1}+b_{2}+b_{3} \\
r=S+v
\end{gathered}
$$

equations (3) and (5) can be written as (see Fig. 2)

\section{Results and Model Analysis}

$$
\frac{d Y}{d t}=\frac{b e X Y}{P}-(u+r) Y
$$

During the first occurrences of the epidemic, we can assume that all members of the community are susceptible, which translates as $X \approx P$. Here, the growth of the infected population $Y(t)$ is then (Cardenas, 2018)

$$
\frac{d Y}{d t}=(b e-[u+r]) Y
$$

On the other hand, calling $T=\frac{u+1}{r}$, the time during which individuals continue to be infected and defining the basic reproductive ratio (Stuart, 2002) for

$$
R_{0}=b e T,
$$

we have 


$$
\frac{d Y}{Y}=\left(b e-\frac{1}{T}\right) d t
$$

where

$$
Y(t)=Y(0) e^{\frac{\left(R_{0}-1\right)}{T} t},
$$

here, $Y(0)$ is the initial number of infected individuals at the time $t=0$ (Fajardo, 2017). We can clearly see that $R_{0}>1$ implies persistence of the epidemic, while $R_{0}<1$ means that $Y(t) \rightarrow 0$.

Now, knowing the time this population takes to double $t_{d}$, we can estimate by Eq. (20) the basic reproductive rate:

$$
R_{0}=1+\frac{(\ln 2) T}{t_{d}}
$$

since $Y(t)=2 Y(0)$. Here, infectivity $\mathrm{b}$ can be evaluated by just matching the Eqs. (19) and (21)

$$
b=\frac{1}{T e}\left(R_{0}\right)
$$

Now, we analyze the phenomenon and the effects of immigration towards the homosexual community (Schalk, 2018). In Eq. (1):

$$
\frac{d P}{d t}=A-u P-a S=A-(a S+u P)
$$

where,

- $u P$ is the number of individuals per natural death.

- $a S$ is the number of individuals died from AIDS.

- $A$ is the number of individuals entering the homosexual community.

- If $A>(a S+u P)$, then $P$ increases over time.

- If $A<(a S+u P)$, then $P$ decreases.

- If $A=(a S+u P)$, then $P$ remains in steady state,

$$
P^{*}=\frac{A-a S}{u}
$$

In the case of incidents, a decrease in be such that $b e<u+r$, will imply that the number of HIV-infected will begin to decline at an early stage before a greater proportion of individuals are infected. If it happens that $b_{i} e<u+r$, then the incidences of HIV-AIDS will decrease.

We can notice that the derivative $P^{\prime}$ in Eq.(1) does not directly depend on $b_{i}$ and $e$. Thus, $P(t)$ will not be affected by a change in $b_{i} e$. According to Eq.(1), a decline in HIV incidence, $y_{i}(t)$ will result in a growth of the $P^{\prime}$ derivative, as the probability of $S$ occurring will be decreasing. Therefore, through a social intervention, it is possible that this derivative is positive (if it is not before), in this form, $P(t)$ and the number of susceptible $X(t)$ will grow at a greater rate (Kurtis, 2016).

Now, let's look at AIDS patients. In Figure 1, it can be seen that every HIV-infected individual will spend time in one of the $Y_{i}$ compartments (Sierra, 2005). Then, he either goes to the natural death compartment or becomes HIV-positive or arrives at the AIDS department, after transiting for $W$ or $U$.

Let's consider Eq. (8):

$$
\frac{d S}{d t}=\left(r y_{2}+c U\right)-(u+a) S,
$$

where we observe the following possible effects:

- Population is expected to increase over time when $\left(r y_{2}+c U\right)>(u+a) S$.

- $S$ is expected to decrease over time when $\left(r y_{2}+c U\right)<(u+a) S$.

- $S$ will remain constant when $\left(r y_{2}+c U\right)=(u+a) S$, which implies that

$$
S^{*}=\frac{r y_{2}+c U}{\mathrm{u}+\mathrm{a}}
$$




\section{Conclusion}

In this work, although many simplifications are presented, it presents very interesting details associated with the patterns of variation over time and exhibits oscillations cushioned by decreasing amplitudes of oscillations towards a stable equilibrium, which explains the dissipation of the incidence.

It is important to emphasize that medical intervention involves the treatment of HIV/AIDS victims for opportunisticsymptomatic diseases. These interventions affect the $r$ and a parameters of our model. The social intervention is through educational programs that tend to promote the reduction in the parameter $b$ (infectibility) through the use of condoms and the parameter $e$ (average number of new sexual partners per unit). Medical intervention tends to prolong the incubation period $\frac{1}{r}$ and reduce the ratio $a$.

\section{Acknowledgements}

We would like to thank the referee for his valuable suggestions that improved the presentation of this paper and our gratitude to Department of Mathematics of the Universidad Tecnologica de Pereira (Colombia), the group GEDNOL and Fundación Universitaria del Área Andina - Pereira.

\section{References}

Bailey, N. (1975). The mathematical theory of infectious diseases. Ed. Griffin. London.

Cárdenas, P. (2017). An iterative Method for Solving Delay Differential Equations Applied to Biological Models. Applied Mathematical Sciences, 11, 1287-1295. https://doi.org/10.12988/ams.2017.7399

Cárdenas, P. (2018). Some Biomathematical Models Applying Adomian Method, International Journal of ChemTech Research, 11, 239-246. https://doi.org/10.20902/IJCTR.2018.110729

Fajardo, D. (2017). The emergence and evolution of the research fronts in HIV-AIDS research, Plos-One, 12, 1-13. https://doi.org/10.1371/journal.pone.0178293

Kurtis, M. (2016). The effects of food web structure on ecosystem function exceeds those of precipitation, Journal of Animal Ecology, 85, 1147-1160. https://doi.org/10.1111/1365-2656.12538

Massad, E. (1989). A homogeneously mixing population model for the AIDS epidemic, Mathematical and Computer Modelling, 12, 89-96.

Stuart, F. (2002). Trophic Dynamics. Principles of Terrestrial Ecosystem Ecology, 250-251.

Schalk, C. (2018). Squamates as prey: Predator diversity patterns and predator-prey size relationships. Food Webs. https://doi.org/10.1016/j.fooweb.2018.e00103

Sierra, S. (2005). Basics of the virology of HIV-1 and its replication, Journal of clinical virology, 34, 233-244.

\section{Copyrights}

Copyright for this article is retained by the author(s), with first publication rights granted to the journal.

This is an open-access article distributed under the terms and conditions of the Creative Commons Attribution license (http://creativecommons.org/licenses/by/3.0/). 